\title{
Kidney Cancer
}

\section{Disagreement in risk groups for metastatic renal cancer}

Tobias Klatte ${ }^{1,2}$ and Grant D Stewart ${ }^{2,3}$

Author ORCIDs:

T Klatte: 0000-0002-4392-6861

GD Stewart: 0000-0003-3188-9140

${ }^{1}$ Department of Urology, Royal Bournemouth and Christchurch Hospitals NHS

Foundation Trust, Bournemouth, UK

${ }^{2}$ Department of Surgery, University of Cambridge, Cambridge, UK

${ }^{3}$ Department of Urology, Cambridge University Hospitals NHS Foundation Trust, Cambridge, UK

\section{Competing interests}

The authors declare no competing interests.

\section{Corresponding author}

Email: tobias.klatte@gmx.de 
In patients with metastatic renal cell carcinoma, risk stratification according to the Memorial Sloan-Kettering Cancer Center or the International Metastatic Renal Cell Carcinoma Database Consortium classification systems is a crucial part of clinical assessment and essential for guiding management. New research has now demonstrated that disagreement in risk-group classification is common and prognostically relevant.

Refers to Okita, K. et al. Impact of disagreement between two risk group models on prognosis in patients with metastatic renal cell carcinoma. Clin Genitourin. Cancer https://dx.doi.org.10.1016/j.clgc.2019.01.006 (2019). 
In patients with newly diagnosed metastatic renal cell carcinoma (mRCC), risk stratification is a crucial part of clinical assessment and essential for guiding management. Major clinical trials defining current treatment paradigms have relied on the Memorial Sloan-Kettering Cancer Center $(\mathrm{MSKCC})^{1}$ or the International Metastatic Renal Cell Carcinoma Database Consortium (IMDC) ${ }^{2}$ risk-group classifications. Both were established using similar methodology and are fairly comparable with regards to variables and risk-group assignment (Table 1). The MSKCC classification was developed using data from patients treated with cytokines, whereas the IMDC model was established using data from patients treated with targeted therapies. The IMDC classification uses markers of systemic inflammatory response such as neutrophil and platelet count, but the MSKCC classification employs lactate dehydrogenase (LDH) as a marker of cell death. ${ }^{1,2}$ Current guidelines advocate the use of a model but do not recommend one in particular. ${ }^{3}$

Even though treatment decisions are often made on the basis of the assigned riskgroup, both classifications have several shortcomings. First and foremost, concordance indices (i.e. the discrimination to predict survival) are low, ${ }^{2}$ and both models are prognostic but not predictive. They do not consider metastatic site, tumor burden, histological type or molecular markers, which all might improve model discrimination. Furthermore, both classifications are mostly blood based and rely on dichotomized variables, both of which are problematic. Dichotomization of independent variables relies on statistical assumptions that are not reasonable, i.e. that the association with the outcome is flat in each group. The cutpoints used in laboratories are often derived from percentiling the readings of a healthy cohort, but there is no agreement if these cutpoints are valid in patients with mRCC. Finally, dichotomization is completely at odds with the concept of making optimal decisions, which relies on a function that takes all other predictors into consideration. ${ }^{4}$ Finally, 
none of the blood variables currently included in the MSKCC and IMDC system are sensitive for RCC or even for cancer, and the classification is dynamic. Indeed, the risk group into which the patient is classified can change quickly without intervention if blood values are close to the normal range or if performance status is assessed by another person. Additionally, cytoreductive nephrectomy has been shown to result in normalization of neutrophil and platelet counts in the majority of patients. ${ }^{5}$ These factors can all result in a change in risk-group classification and management recommendations.

Little research has been conducted on the agreement in risk-group classification between the MSKCC and IMDC classification systems. Recently, Okita et al. ${ }^{6}$ studied 176 patients with synchronous or asynchronous $\mathrm{mRCC}$ who received first-line sunitinib, sorafenib or axitinib. Although Cohen's k coefficient demonstrated good agreement between both classification systems in general, disagreement was seen in about one-quarter of patients. This involved a change from MSKCC intermediate-risk to IMDC-poor-risk disease or vice versa in $>80 \%$ of reclassified patients, and the majority of changes were related to the neutrophil count. Disagreement was associated with reduced progression-free survival ( $\operatorname{HR} 1.9, P=0.025)$ and overall survival $(\mathrm{HR}=1.75, P=0.028) .{ }^{6}$ Thus, disagreement is common, prognostically relevant and has the potential to influence management.

The CARMENA trial ${ }^{7}$ (NCT00930033) showed that sunitinib alone is not inferior to immediate cytoreductive nephrectomy followed by sunitinib in patients with synchronous metastatic clear-cell RCC. The CARMENA trial relied on the MSKCC classification and stratified disease into intermediate risk and poor risk, which covers nearly $100 \%$ of patients with synchronous $\mathrm{mRCC}$, as patients with this disease cannot be stratified into the favorable-risk group at the time of presentation. Only patients whose disease does not require systemic treatment for at least 1 year can 
be classified into the favorable-risk group, which can only be judged retrospectively. On the basis of prospective and retrospective evidence, the European Association of Urology Guidelines ${ }^{3}$ recommend not to perform cytoreductive nephrectomy in patients classified as having MSKCC poor-risk disease, not to perform immediate cytoreductive nephrectomy in patients classified as having MSKCC intermediate-risk disease with an asymptomatic primary tumor who require systemic treatment and to perform immediate cytoreductive nephrectomy in patients with good performance status and MSKCC favorable- or intermediate-risk disease who do not require immediate systemic treatment. Thus, cytoreductive nephrectomy can be offered to a minority of patients with MSKCC intermediate-risk disease and the chance of these patients having IMDC poor-risk disease is $25-30 \%$ according to Okita et al. ${ }^{6}$ The results of CARMENA are not applicable to the IMDC classification owing to the differences between the classification systems, but patients with IMDC poor-risk disease are unlikely to benefit from surgery. ${ }^{8}$

Currently, recommended systemic first-line treatments for mRCC are sunitinib, pazopanib, cabozantinib, or nivolumab plus ipilimumab. ${ }^{3}$ Trials leading to approval of these agents differed with regards to inclusion criteria and risk-group classification. On the basis of the results of the CheckMate 214 trial $^{9}$ (NCT02231749), nivolumab plus ipilimumab has now become standard fist-line treatment for patients with IMDCintermediate or poor-risk disease. The results of this trial are not applicable to patients with favorable-risk disease, for which sunitinib or pazopanib remain the preferred options. The original sunitinib trial $\left(\right.$ NCT00083889 $\left.{ }^{10}\right)$ included patients of all prognostic groups, but risk group was assigned according to the MSKCC classification system. As a progression-free survival benefit of sunitinib was seen in all subgroups of patients ${ }^{10}$, and nivolumab plus ipilimumab was superior to sunitinib for IMDC intermediate-risk and poor-risk disease ${ }^{9}$, disagreement between MSKCC 
and IMDC has no effect on guideline-recommended first-line treatment. However, importantly, nearly $50 \%$ of patients with IMDC poor-risk disease could be reclassified to the MSKCC intermediate-risk group, whereas $50 \%$ would remain in the MSKCC poor-risk group according to the results of Okita and colleagues. ${ }^{6}$ Evidence is insufficient to support use of sunitinib in patients with MSKCC poor-risk disease and, therefore, it might not be an alternative option for these patients if nivolumab plus ipilimumab is not feasible or safe.

In summary, risk group disagreement between MSKCC and IMDC is common and generally associated with poor prognosis, but the guideline-recommended first-line treatment remains unaltered in the vast majority of patients. Group disagreement could be routinely assessed as prognostic factor in clinical practice and might improve discrimination of current models. 
Table 1. MSKCC and IMDC risk classifications for $\mathrm{mRCC}$

\begin{tabular}{|c|c|c|}
\hline Variable & Risk factor & Risk group \\
\hline MSKCC & & \\
\hline $\begin{array}{l}\text { Time from initial } \\
\text { diagnosis to systemic } \\
\text { treatment }\end{array}$ & $<1$ year & \multirow[t]{5}{*}{$\begin{array}{l}\text { 0 RF: favorable } \\
\text { 1-2 RF: intermediate } \\
\text { 3-5 RF: poor }\end{array}$} \\
\hline Hemoglobin & $<$ lower limit of normal range & \\
\hline Corrected calcium & $>10 \mathrm{mg} / \mathrm{dl}$ & \\
\hline Lactate dehydrogenase & $>1.5 \times$ upper limit of normal range & \\
\hline $\begin{array}{l}\text { Karnofsky Performance } \\
\text { Status }\end{array}$ & $<80 \%$ & \\
\hline IMDC & & \\
\hline $\begin{array}{l}\text { Time from initial } \\
\text { diagnosis to systemic } \\
\text { treatment }\end{array}$ & $<1$ year & \multirow[t]{6}{*}{$\begin{array}{l}\text { 0 RF: favorable } \\
\text { 1-2 RF: intermediate } \\
\text { 3-6 RF: poor }\end{array}$} \\
\hline Hemoglobin & $<$ lower limit of normal range & \\
\hline Corrected calcium & >upper limit of normal range & \\
\hline Neutrophil count & >upper limit of normal range & \\
\hline Platelet count & >upper limit of normal range & \\
\hline $\begin{array}{l}\text { Karnofsky Performance } \\
\text { Status }\end{array}$ & $<80 \%$ & \\
\hline
\end{tabular}

IMDC, International Metastatic Renal Cell Carcinoma Database Consortium; mRCC, metastatic renal cell carcinoma; MSKCC; Memorial Sloan-Kettering Cancer Center; RF, risk factor. 


\section{References}

1. Motzer, R. J., Bacik, J., Murphy, B. A., Russo, P. \& Mazumdar, M. Interferon-alfa as a comparative treatment for clinical trials of new therapies against advanced renal cell carcinoma. J Clin Oncol 20, 289-296 (2002).

2. Heng, D. Y. C. et al. Prognostic factors for overall survival in patients with metastatic renal cell carcinoma treated with vascular endothelial growth factortargeted agents: results from a large, multicenter study. J Clin Oncol 27, 57945799 (2009).

3. Ljungberg, B. et al. EAU Guidelines on Renal Cell Carcinoma. (2019). Available at: http://uroweb.org/guideline/renal-cell-carcinoma/.

4. Harrell, Frank E. Problems Caused by Categorizing Continuous Variables. Available at: http://biostat.mc.vanderbilt.edu/wiki/Main/CatContinuous. (Accessed: 16th February 2019)

5. Noe, A. et al. Comparison of pre-treatment MSKCC and IMDC prognostic risk models in patients with synchronous metastatic renal cell carcinoma treated in the era of targeted therapy. World J Urol 34, 1067-1072 (2016).

6. Okita, K. et al. Impact of disagreement between two risk group models on prognosis in patients with metastatic renal cell carcinoma. Clin Genitourin Cancer (2019). doi:10.1016/j.clgc.2019.01.006

7. Méjean, A. et al. Sunitinib Alone or after Nephrectomy in Metastatic Renal-Cell Carcinoma. N. Engl. J. Med. 379, 417-427 (2018).

8. Heng, D. Y. C. et al. Cytoreductive nephrectomy in patients with synchronous metastases from renal cell carcinoma: results from the International Metastatic Renal Cell Carcinoma Database Consortium. Eur. Urol. 66, 704-710 (2014). 
9. Motzer, R. J. et al. Nivolumab plus Ipilimumab versus Sunitinib in Advanced Renal-Cell Carcinoma. N. Engl. J. Med. 378, 1277-1290 (2018).

10. Motzer, R. J. et al. Sunitinib versus interferon alfa in metastatic renal-cell carcinoma. N. Engl. J. Med. 356, 115-124 (2007). 